\title{
IMAGE DENOISINg USING SURE-BASED ADAPTIVE THRESHOLDING IN DIRECTIONLET DOMAIN
}

\author{
Sethunadh $\mathrm{R}^{1}$ and Tessamma Thomas ${ }^{2}$ \\ ${ }^{1}$ Vikram Sarabhai Space Centre (VSSC), Indian Space Research Organisation (ISRO), \\ Trivandrum, India \\ r_sethunadh@vssc.gov.in \\ ${ }^{2}$ Cochin University of Science \& Technology (CUSAT), Kochi, India \\ tess@cusat.ac.in
}

\begin{abstract}
The standard separable two dimensional wavelet transform has achieved a great success in image denoising applications due to its sparse representation of images. However it fails to capture efficiently the anisotropic geometric structures like edges and contours in images as they intersect too many wavelet basis functions and lead to a non-sparse representation. In this paper a novel de-noising scheme based on multi directional and anisotropic wavelet transform called directionlet is presented. The image denoising in wavelet domain has been extended to the directionlet domain to make the image features to concentrate on fewer coefficients so that more effective thresholding is possible. The image is first segmented and the dominant direction of each segment is identified to make a directional map. Then according to the directional map, the directionlet transform is taken along the dominant direction of the selected segment. The decomposed images with directional energy are used for scale dependent subband adaptive optimal threshold computation based on SURE risk. This threshold is then applied to the sub-bands except the LLL subband. The threshold corrected sub-bands with the unprocessed first sub-band (LLL) are given as input to the inverse directionlet algorithm for getting the de-noised image. Experimental results show that the proposed method outperforms the standard wavelet-based denoising methods in terms of numeric and visual quality.
\end{abstract}

\section{KEYWORDS}

Undecimated directionlet transform, Directional map, Denoising, SURE threshold.

\section{INTRODUCTION}

Image noise is an undesirable by-product of image acquisition or transmission. Denoising is one of the important pre-processing steps in various image processing and analysis applications. The main aim of image denoising is to remove noise while preserving the important signal features. Noise reduction techniques are conceptually similar regardless of the image being processed; however a prior knowledge of the characteristics of an expected image can govern the implementations of these techniques. A number of denoising methods have been proposed in literature for removing various types of noises such as Gaussian, Speckle, Salt \& Pepper etc. These include linear and non-linear techniques. Noise having Gaussian-like distribution is very often encountered in real-world images. The zero mean property of the Gaussian distribution allows such noise to be removed by locally averaging pixel values. Conventional linear filters such as arithmetic mean filter and Gaussian filter smooth noises effectively but distort edges and contours [1]. The Wiener filter is the mean square error-optimal stationary linear filter for images degraded by additive noise and blur. A common drawback of the practical use of this method is that they usually require some 'a priori' knowledge about the spectra of noise and the original signal. Unfortunately, such information is very often not available. This makes the linear or DOI : $10.5121 /$ sipij.2012.3606 
Signal \& Image Processing : An International Journal (SIPIJ) Vol.3, No.6, December 2012

spatial techniques less attractive for image denoising. Alternatively non-linear methods were proposed for denoising. They are mostly based on multi-resolution analysis using wavelet transform [2, 3]. In the wavelet domain, the noise is uniformly spread throughout the coefficients, while most of the image information is concentrated in the few largest ones due to its sparse representation. The most straightforward way of distinguishing information from noise in the wavelet domain consists of thresholding the wavelet coefficients [4, 5]. A wavelet coefficient is compared with a given threshold and is set to zero if its magnitude is less than the threshold; otherwise it is kept or modified depending on hard or soft thresholding schemes. Since 2-D Wavelet is tensor product of 1-D Wavelet, it has only three directions, viz. vertical, horizontal and diagonal. So 2-D Wavelet is effective at approximating point singularities than line singularities like edges. The tensor product wavelet do not adapt to the boundaries or edges, due to isotropic scaling of its basis functions. Therefore a more effective basis for real-world images with edges and curves is required for making the signal to concentrate on fewer coefficients after transformation.

To make image representation effective for denoising applications, it should be based on a local, directional and multi resolution expansion. Towards this several adaptive schemes based on bandelets [6] and wedgelets [7] and non-adaptive schemes based on curvelets [8] and contourlets $[9,10,11,12]$ have been proposed. These methods build dictionaries of anisotropic oriented basis functions that provide a sparse representation of edges and contours in images. These geometrical transforms have good directionality than wavelet transform. However, these transforms often require oversampling, have higher computational complexity when compared to the standard WT, and require non-separable convolution and filter design due to the non-rectangular division of frequency spectrum. Furthermore, in some of these transforms like curvelets the design of the associated filters is performed in the continuous domain and this makes it difficult to use them directly on discrete images. Also some other directional transforms like directional filter bank (DFB) lacks in multi-scale feature even if it can provide perfect reconstruction and cascade structures [13]. The DFB combined with the Laplacian Pyramid [14] can overcome this difficulty. But these methods are computationally complex and the design of the associated 2-D filter-banks is often challenging and involved.

The directionlet transform was proposed by Vladen et al as an anisotropic perfect reconstruction and critically sampled basis functions with directional vanishing moments along any two directions [15]. It retains the simplicity of 1-D processing and filter design from the standard separable 2-D WT. It has good approximation properties as compared to the approximation achieved by the other over complete transform constructions and is superior to the performance of the standard separable 2-D WT while having the same complexity. Eventhough directionlet was designed to provide image compression [16], it can be used in collaboration with directional energy for image enhancement purpose also. As far as image compression is concerned, decimation plays a central role. But whenever it is employed for enhancement and recognition purpose, decimation becomes a problem. Here in the proposed work an undecimated version of directionlet transform is used for denoising.

After making an effective sparse representation of image the most straight forward way of distinguishing information from noise is the thresholding of the sparsely represented coefficients. Even if thresholding is a non linear technique, it is very simple because it operates on one sample at a time. There are ample of literature available on finding out an effective threshold. Of the various thresholding strategies, soft-thresholding is the most popular and has been theoretically justified by Donoho and Johnstone [17]. These authors have shown that the shrinkage rule is near-optimal in the minimax (minimum of maximum mean square error) sense and provided the expression of the optimal threshold called universal threshold as a function of the noise power, when the number of samples is large. The use of the universal threshold to denoised images in the wavelet domain is known as VisuShrink. For image denoising, however, VisuShrink is known to 
Signal \& Image Processing : An International Journal (SIPIJ) Vol.3, No.6, December 2012

yield overly smoothed images. This is because its threshold value, $\sqrt{2 \sigma^{2} \log N}$, can be unwarrantedly large due to its dependence on the number of samples $\mathrm{N}$, which is very high for typical test images. Yet, despite its theoretical appeal, minimax is different from mean-squared error (MSE) as a measure of error. For denoising applications with known noisy function, it is often ideal to search for the optimal minimum mean-square error risk estimate using a priori information. Thus Donoho and Johnstone proposed an optimal threshold value by minimizing Stein's unbiased risk estimator (SURE) [18]. SURE risk is a very good estimation of the true risk when the true function is not known $[19,20]$. Later a hybrid approach between the universal threshold and SURE threshold was proved to be more efficient and is known as SURE shrink [21]. Later many authors have proposed different image denoising algorithms based on wavelet coefficient thresholding [22, 23, 24 and 25].

Here we propose an image denoising method based on an optimized hybrid scale dependent adaptive thresholding scheme using SURE risk in directionlet domain. We show that the proposed method provides much better perceptual and numerical image estimates.

The paper is organised as follows. In section 2, the theoretical concepts of Directionlet Transform are presented. Section 3 explains the SURE thresholding scheme in wavelet domain. The proposed denoising algorithm based on directionlet is presented in section 4. Experimental results with different test images and the comparison with wavelet based denoising scheme are given in section 5 .

\section{DireCTIONLET TRANSFORM}

The standard Wavelet Transform (WT) is an efficient tool for analysing one dimensional signal. However, for 2-D signals like images it is inefficient due to the spatial isotropy of its construction. In 2-D WT the number of 1-D transforms including filtering and sub-sampling operations along the horizontal and vertical directions is the same at each scale (Figure 1(a)). This isotropic transform cannot properly capture the anisotropic discontinuities present in images. This is because the directions of the transforms and discontinuities in images are not matched and the transform fail to provide a compact representation of two dimensional signals. The standard WT considers only vertical and horizontal directions and number of filtering in both these directions is equal. Because of this, a separable 2D wavelet transform applied on natural images with edges in orientations other than horizontal and vertical, results in too many non-zero coefficients. Here the 1-D discontinuities like edges or contours of highly anisotropic objects cannot be properly captured by the isotropic WT. Many wavelets intersect the discontinuity and this leads to many large magnitude coefficients (see Figure 1(c)).

In the anisotropic wavelet transform (AWT $\left.\left(n_{1}, n_{2}\right)\right)$, the number of transforms applied along the horizontal and vertical directions is unequal, that is there are $n_{1}$ horizontal and $n_{2}$ vertical transforms at a scale, where $n_{1}$ is not necessarily equal to $n_{2}$. The iteration process is continued in the low sub-band, like in the standard wavelet transform (Figure 1(b)). The anisotropic wavelet transform can be implemented as a stage transformation. At the first stage the rows of the image to be transformed are low pass (L) and high pass $(\mathrm{H})$ filtered and down sampled by two. In the next step each column of the row filtered image is again low pass $(\mathrm{L})$ and high pass $(\mathrm{H})$ filtered and down sampled by two. The output of this will have four sub band images labeled as LL, HL, LH and HH. As a next step each row of these sub band images are again low pass (L) and high pass $(\mathrm{H})$ filtered and down sampled by two. This will produce eight bands viz. HHH, HHL, HLH, HLL, LHH, LHL, LLH, and LLL as shown in Figure 2. Now the LLL subband image goes through the same process of filtering and down sampling to form the next stage of the structure. The anisotropic ratio $\rho=n_{1} / n_{2}$ determines elongation of the basis functions of the AWT $\left(n_{1}, n_{2}\right)$. When $n_{1}=2, n_{2}=1$, the AWT $(2,1)$ produces eight bands as shown in Figure 2. The AWT can 
trace the discontinuity efficiently with fewer significant coefficients compared with standard WT (see Figure 1(d)).

The main problem with AWT is directional interaction. The lattice based transform can avoid the directional interaction. Here the discrete space is first partitioned using integer lattices before performing 1-D filtering along lines across the lattice. Any integer lattice $\Lambda$ is a sub-lattice of the cubic lattice $Z^{2}$. Here the lattice $\Lambda$ can be represented by a non-unique generator matrix $\mathrm{M}_{\Lambda}$.

$\mathbf{M}_{\Lambda}=\left[\begin{array}{ll}a_{1} & b_{1} \\ a_{2} & b_{2}\end{array}\right]=\left[\begin{array}{l}d_{1} \\ d_{2}\end{array}\right]$

where, $\mathrm{a}_{1}, b_{1}, a_{2}, b_{2} \in Z$.

The linear combination of two linearly independent integer vectors $d_{l}$ and $d 2$ will form the points of the lattice $\Lambda$. The cubic lattice $\mathrm{Z}^{2}$ can be partitioned into $\left|M_{\Lambda}\right|$ cosets of the lattice $\Lambda$. The filtering and sub sampling operations are applied on the pixels along the vector $d_{l}$ (transform direction) in each of the cosets separately. Since these operations are applied in each cosets separately, the pixels retained after this are clustered along the vector $d_{2}$ (alignment direction). This type of lattice based transform, which will avoid directional interaction, is called Skewed AWT, S-AWT $\left(\mathrm{M}_{\Lambda}, n_{1}, n_{2}\right)$. The basis functions of S-AWT are called directionlets, which can be effectively used for directional analysis of images. An example of construction of directionlets based on integer lattices is shown in Fig. 3 for pair of direction $\left(45^{\circ},-45^{\circ}\right)$.

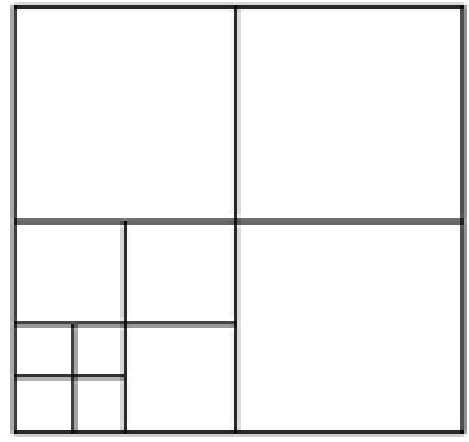

(a)

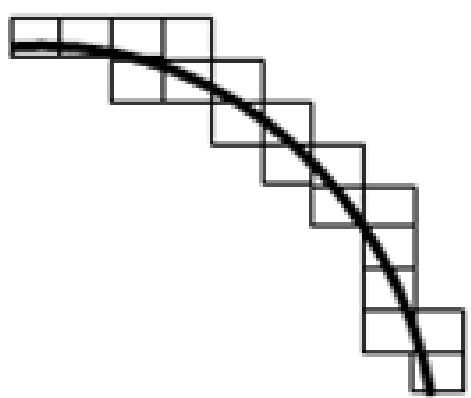

(c)

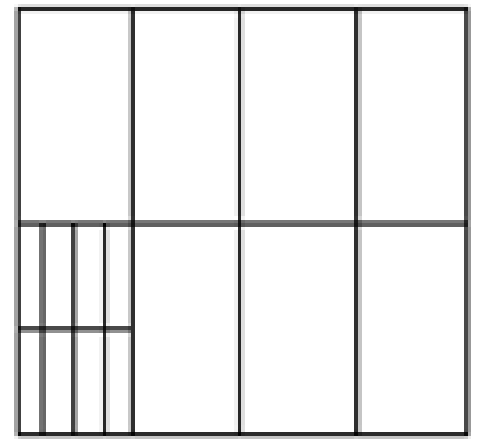

(b)

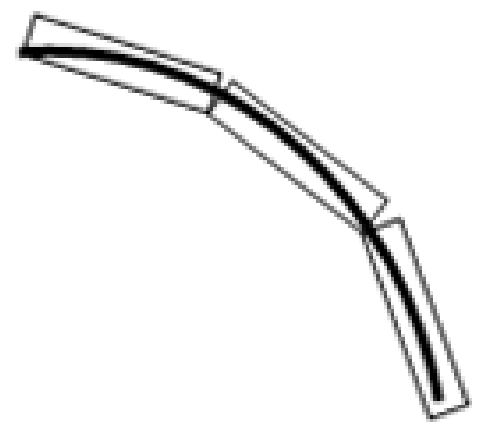

(d)

Figure 1. Frequency decomposition of (a) Standard 2-D Wavelet Transform (b) Anisotropic Wavelet Transform Basis functions of (c) 2-D Wavelet Transform (d) Anisotropic Wavelet Transform. 
Signal \& Image Processing : An International Journal (SIPIJ) Vol.3, No.6, December 2012

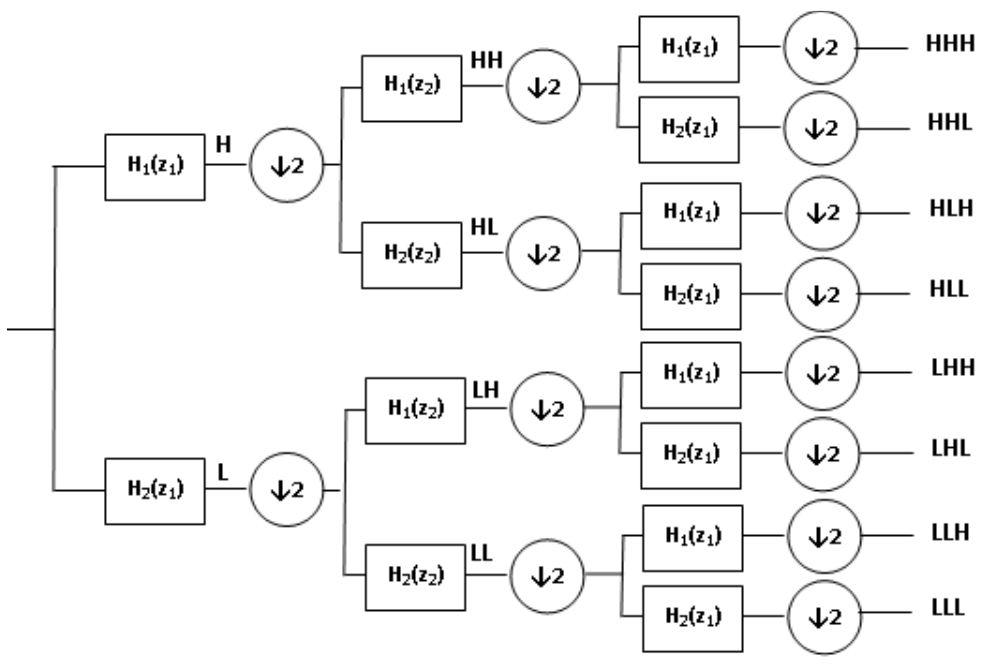

Figure 2. Filtering scheme for the AWT $(2,1)$, where one step of iteration is shown.
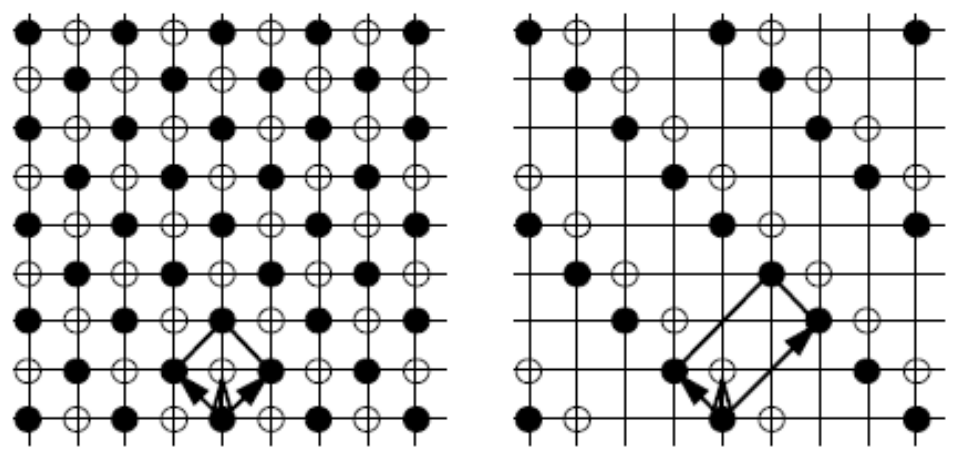

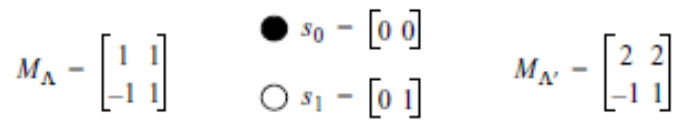

Figure 3. An example of construction of directionlets based on integer lattices for pair of directions $\left(45^{\circ},-45^{\circ}\right)$.

Unlike the discrete version of directionlet transform, which down samples the the approximation coefficients and detail coefficients at each decomposition level, the Undecimated Directionlet Transform (UDT) does not incorporate the down sampling operations. Thus, the approximation coefficients and detail coefficients at each level are having the same length as the original signal. Denoising with the UDT is shift-invariant.

\section{Sure Thresholding In WAVELET Domain}

Suppose that the image $f$, with $\mathrm{N}$ number of pixels, is polluted by independent and identically distributed white Gaussian noise $n$ with mean 0 and variance $\sigma^{2}$, the observed image $y$ is described as:

$$
y=f+n
$$


Signal \& Image Processing : An International Journal (SIPIJ) Vol.3, No.6, December 2012

In denoising applications, the performance is often measured in terms of peak signal-to-noise ratio (PSNR). The aim of image denoising is naturally to maximize the PSNR and, thus, to minimize the minimum mean square error (MSE). Here our objective is to estimate image $f$ with minimum MSE, i.e., to minimize $\boldsymbol{l}_{2}$ risk for a given noisy image, as follows:

$$
R(\hat{f}, f)=\frac{1}{N}\|\hat{f}-f\|^{2}=\frac{1}{N} \sum_{i=0}^{N-1}\left(\hat{f}_{i}-f_{i}\right)^{2}
$$

Here $\hat{f}$ is the estimate of $f$ from $y$. Here mean is used instead of the mathematical expectation, because the optimal solution is desired for each individual noisy function. For the image noise model given in (2), assume

$$
x(y)=\hat{f}(y)-y
$$

where $x(y)$ is a function from $R^{N}$ to $R^{N}$.

Charles Stein [18] introduced a method for estimating the loss $\|\hat{f}-f\|^{2}$ in an unbiased fashion. For a nearly arbitrary, nonlinear, biased estimator one can nevertheless estimate its loss unbiased $[19,20]$. When $x(y)$ is weakly differentiable, then

$$
E_{f}\|\hat{f}(y)-f(y)\|^{2}=N+E_{f}\left\{\|x(y)\|^{2}+2 \nabla_{\mathrm{y}} x(\mathrm{y})\right\}
$$

Where,

$$
\nabla_{\mathrm{y}} x(y)=\sum_{\mathrm{i}=0}^{\mathrm{N}} \frac{\partial x_{\mathrm{i}}}{\partial \mathrm{y}_{\mathrm{i}}}
$$

Now consider the soft threshold estimator

$$
\hat{f}(y)=\eta(y, t)=\operatorname{sign}(y)(|y|-t)_{+}
$$

Where

$$
\begin{gathered}
(|y|-t)_{+}=\max (0,|y|-t) \\
\operatorname{sign}(y)=\frac{1}{1+e^{-y}}
\end{gathered}
$$

$\hat{f}(y)$ is weakly differentiable in Stein's sense, and so we get from (5) that the quantity

$\|\hat{f}(y)-f(y)\|^{2}=N-2 . \neq\left\{i:\left|y_{i}\right| \leq t+\sum_{i=1}^{N}\left(\left|y_{i}\right| \wedge t\right)^{2}\right\}=R_{S}(t)$

This is an unbiased estimator of the risk, $E_{f}\|\hat{f}(y)-f(y)\|^{2}$.

This estimator of risk can be used to select the near optimal threshold as

$$
t_{s}=\underset{t \in\left\{y_{0}, y_{1} . . y_{N-1}\right\}}{\arg \min } R_{S}(t)
$$

Here $t_{s}$ is selected as the value of $y$ which makes the $R_{S}(t)$ minimum. This is a suboptimal threshold for the risk because it is selected within a finite set. 
Signal \& Image Processing : An International Journal (SIPIJ) Vol.3, No.6, December 2012

The wavelet shrinkage method relies on the basic idea that the energy of a function will often be concentrated in a few coefficients in wavelet domain while the energy of noise is spread among all coefficients. Therefore, the nonlinear thresholding function in wavelet domain will tend to keep a few larger coefficients representing the function, while the noise coefficients will tend to reduce to zero. Here the equation for $\boldsymbol{l}_{2}$ risk for a given noisy image can be extended to the wavelet coefficients also provided the transform is orthogonal. Then the risk function given in (3) can be expressed as in wavelet domain [21] as

$$
R(\hat{f}, f)=\frac{1}{N} \sum_{i=0}^{N-1}\left(\hat{f}_{i}-f_{i}\right)^{2}=\frac{1}{N} \sum_{j, k}\left(\hat{F}_{j, k}-F_{j, k}\right)^{2}
$$

Apply the discrete wavelet transform (DWT) to the vector $y$ and obtain the empirical wavelet coefficients $Y_{j, k}$ at scale $j$ where $j=1,2 \ldots . J$. Here $Y_{0, k}$ represents the scaling coefficients and will not be shrunk in the next step. Then apply the soft-thresholding function as given in equation (7), to the empirical wavelet coefficients at each scale $j$ where $j=1,2 \ldots . J$. Then the estimate coefficients $\hat{Y}_{j, k}$ are obtained based on the selected threshold $t=\left[t_{1}, t_{2} \ldots t_{J}\right]^{T}$. Note $t_{j}$ is the threshold for wavelet coefficients at scale $j$. Here $t$ is selected for each scale as the value of the wavelet coefficient at that scale which makes the SURE risk $R_{S}(t)$ minimum. This is done for each detail subband. So this is a scale dependent sub band adaptive threshold. Now take the inverse DWT on threshold applied wavelet coefficients and obtain the estimate of the function.

\section{DENOISING AlgorithM IN DIRECTIONLET DOMAIN}

We present a novel denoising scheme which is in line with the 3-band 2-D wavelet transform based schemes presented in previous section with several modifications due to the implementation of directionlet transform. As in any transform based denoising schemes, the directionlet based denoising also involves mainly three steps. First transform the input image using an orthogonal transform, then threshold the transform coefficients using a non-linear algorithm and finally reconstruct the image using the modified coefficients. The effectiveness of a denoising algorithm basically depends on two factors- one is the efficient representation of the image to be denoised using a local, directional and multi resolution expansion and second is the efficient computation of an optimal threshold. Here the first requirement is met by using a locally adaptive directionlet transform and the second by optimal threshold computation using SURE risk.

The edge information in images has great influence on human visual effect and reflects the main direction of texture for the most part. The information on direction of texture with edge information in an image can greatly improve the precision of estimation of correct threshold. Thus it is important to identify the dominant direction of texture before thresholding. In the case of images, the directional information varies over space. Thus, directionality can be considered as a local feature, defined in a small neighborhood. Therefore, to extract directional variations of an image it has to be analysed locally. This necessitates the need for spatial segmentation of image into smaller segments before identifying the dominant directions for that segment. Then the transform directions are adapted independently in each segment based on the identified dominant directions allowing for more efficient capturing of geometrical information. In the proposed scheme the input image is subdivided into patches of size $16 \times 16$ before taking the directionlet transform. To avoid a blocking effect in the transform caused by many small segments, the pixels from the neighbour segments are used for filtering across the segment borders.

Even though the construction of directionlets allow for anisotropy and directional vanishing moments along any two directions with rational slopes, the transform direction pairs are taken only from the set $D=\left\{\left(0^{\circ}, 90^{\circ}\right),\left(0^{\circ}, 45^{\circ}\right),\left(0^{\circ},-45^{\circ}\right),\left(90^{\circ}, 45^{\circ}\right),\left(90^{\circ},-45^{\circ}\right)\right\}$ so that the cubic 
Signal \& Image Processing : An International Journal (SIPIJ) Vol.3, No.6, December 2012

lattice is not divided into more cosets. The best pair of directions $d_{n}^{*} \in D$ is chosen for each segment indexed by $n$ as:

$$
d_{n}^{*}=\underset{d \in D}{\arg \min } \sum_{i}\left|W_{n, i}^{d}\right|^{2}
$$

where the wavelet coefficients $W_{n, i}^{d}$ are produced by applying directionlets to the nth segment along the pair $d$ of directions. The assigned pair of transform directions of each patch across the input image forms a directional map [16] of that image and provides the best matching between transform and locally dominant directions for that segment. For segments with no apparent dominant directions, the pair $(0,90)$ is assigned by default to smooth segments for the reason of simplicity of implementation of the directionlet.

After identifying the dominant direction in each spatial segment, the directionlet coefficients corresponding to the dominant directions are selected for thresholding. In the directionlet decomposition, the subbands $H H H_{k}, H H L_{k}, H L H_{k}, H L L_{k}, L H H_{k}, L H L_{k}$ and $L L H_{k}, k=1,2,3 \ldots J$ are called the details, where $k$ is the scale, with $J$ being the largest (or coarsest) scale in the decomposition. The subband $L_{L L}$ is the low resolution residual. Like wavelet transform, the directionlet transform is also orthogonal and the coefficients in the subbands are independent and identically distributed with zero mean and variance. Thus the image noise model presented in section 3 is applicable for directionlets also. The directionlet coefficient $Y_{i j}$ from the detail subbands are used for optimal threshold computation with soft threshold function to obtain $\hat{X}_{\mathrm{ij}}$. The denoised estimate is then $\hat{f}=D^{-1} \hat{X} \mathrm{ij}$, where $D^{-1}$ is the inverse directionlet transform operator. Figure 4 illustrates the block diagram of the proposed denoising scheme.

Here the undecimated version of directionlet transform (UDT) is used which does not incorporate the down sampling operations. Thus, the approximation coefficients and detail coefficients at each level are having the same length as the original signal. Denoising with the UDT is shiftinvariant. The denoising result of the undecimated directionlet transform has a better balance between smoothness and accuracy than its discrete version.

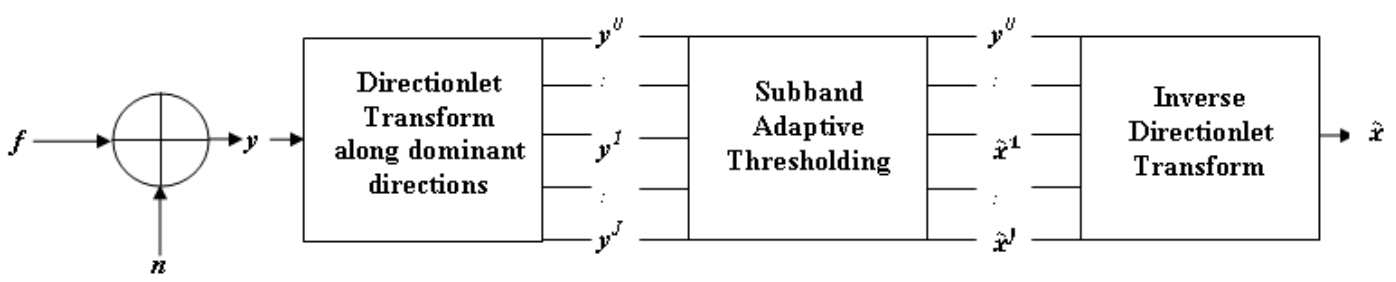

Figure 4. Principle of Directionlet based denoising

The full image denoising algorithm proposed in the paper can be summarised as follows:-

\section{Step 1: Directionlets and Directional Map}

- The noisy image is first divided into spatial segments of smaller size, say 16 by 16 .

- Apply directionlet transform to segments along the pair of directions $D=\left\{\left(0^{\circ}, 90^{\circ}\right),\left(0^{\circ}, 45^{\circ}\right),\left(0^{\circ},-45^{\circ}\right),\left(90^{\circ}, 45^{\circ}\right),\left(90^{\circ},-45^{\circ}\right)\right\}$

- Compute optimal pair of direction using equation (13)

- Compute the multi scale directionlet transform along the dominant direction 
Signal \& Image Processing : An International Journal (SIPIJ) Vol.3, No.6, December 2012

\section{Step 2: Threshold Computation}

- For each subband, except the $L L L_{j}$ subband, compute the SURE threshold using the equation (11) which minimizes the SURE risk

- Apply the computed threshold to the subband using soft thresholding rule to estimate the best value for the noise-free coefficients

\section{Step 3: Reconstruction}

- Reconstruct the image from the above processed sub-bands and the low-pass residual $\left(L L L_{j}\right)$ using inverse directionlet transform and the direction map to obtain the denoised image.

\section{RESULTS AND DISCUSSIONS}

Standard grey images of size $256 \times 256$ were used for evaluating the performance of the developed algorithm. The test images were contaminated with simulated zero mean white Gaussian noise with three different power levels, $\boldsymbol{\sigma}=10,15,20$. The performance of the new method was compared with discrete wavelet based soft thresholding (WST) and hard thresholding schemes (WHT). In our method as well as in DWT based methods we used the popular Haar wavelet. The performance improvement was quantified in terms of Peak Signal to Noise Ratio (PSNR) and edge preservation parameter $\beta$. PSNR was obtained by using the following formula:

$$
P S N R=10 \log _{10}\left(\frac{R^{2}}{M S E}\right)
$$

where $R$ is the maximum fluctuation in the denoised image and MSE is representing the Mean Square Error between the denoised image $I_{d e n}$ and the original image (before adding noise) $I_{\text {org }}$, which was computed using the following equation:

$$
M S E=\frac{\sum_{i, j}\left(I_{d e n}(i, j)-I_{\text {org }}(i, j)\right)^{2}}{M x N}
$$

where $\mathrm{M}$ and $\mathrm{N}$ are the size of the images.

$\beta$ parameter which assesses the quality of the edge preservation was computed by using the following formula:

$$
\beta=\frac{\sum_{i, j}\left(\Delta I_{\text {den }}-\Delta \overline{I_{\text {den }}}\right)\left(\Delta I_{\text {org }}-\Delta \overline{I_{\text {org }}}\right)}{\sqrt{\sum_{i, j}\left(\Delta I_{\text {den }}-\Delta \overline{I_{\text {den }}}\right)^{2} \sum_{i, j}\left(\Delta I_{\text {org }}-\Delta \overline{I_{\text {org }}}\right)^{2}}}
$$

where $\Delta I_{\text {den }}$ and $\Delta I_{\text {org }}$ are the high pass filtered output of $I_{\text {den }}(i, j)$ and $I_{\text {org }}(i, j)$ using Laplacian filter. If the edges are optimally preserved, then the value of $\beta$ will be close to one.

The denoising process was performed over ten different noise realizations for each standard deviation and the resulting PSNRs and $\beta$ averaged over these ten runs. Table I and Table II summarize the relative PSNR results and $\beta$ of wavelet based and the proposed schemes for Lena and house images. A visual comparison of Lena image is given in Figure 5 for $\sigma=25$. The proposed method outperforms the other ones for both the test images. Also the visual quality of the denoised images is evidently better because of sharper edges and texture. We can notice that our method does not produce any artifacts while it is able to well preserve the features of the original image. The performance improvement is basically due to the locally adaptive directional decomposition of image before applying the optimal threshold. 
Signal \& Image Processing : An International Journal (SIPIJ) Vol.3, No.6, December 2012

Table I. PSNR (dB) * values for the different denoising algorithms of Lena \& House (256x256) images

\begin{tabular}{|c|c|c|c|c|}
\hline \multicolumn{5}{|c|}{ Lena } \\
\hline \multirow{2}{*}{ Variance } & Noise PSNR & WST & WHT & Directionlet \\
\hline 5 & 34.24 & 35.61 & 36.48 & 37.71 \\
10 & 28.38 & 30.57 & 32.02 & 33.86 \\
25 & 20.13 & 25.03 & 26.57 & 27.99 \\
\hline \multicolumn{5}{|c|}{ House } \\
\hline Variance & Noise PSNR & WST & WHT & Directionlet \\
\hline 5 & 34.22 & 36.81 & 38.46 & 39.78 \\
25 & 28.32 & 31.81 & 33.82 & 36.98 \\
& 20.17 & 25.96 & 27.41 & 29.12 \\
\hline
\end{tabular}

* Average of ten readings

Table II. $\beta *$ values for the different denoising algorithms of Lena \& House $(256 \times 256)$ images

\begin{tabular}{|c|c|c|c|}
\hline \multicolumn{3}{|c|}{ Lena } \\
\hline Variance & WST & WHT & Directionlet \\
\hline 5 & 0.698 & 0.771 & 0.823 \\
10 & 0.512 & 0.557 & 0.612 \\
25 & 0.221 & 0.301 & 0.392 \\
\hline \multicolumn{3}{|c|}{ House } \\
\hline \multirow{2}{*}{ Variance } & WST & WHT & Directionlet \\
\hline 5 & 0.782 & 0.826 & 0.928 \\
10 & 0.524 & 0.628 & 0.796 \\
25 & 0.301 & 0.372 & 0.467 \\
\hline
\end{tabular}

* Average of ten readings
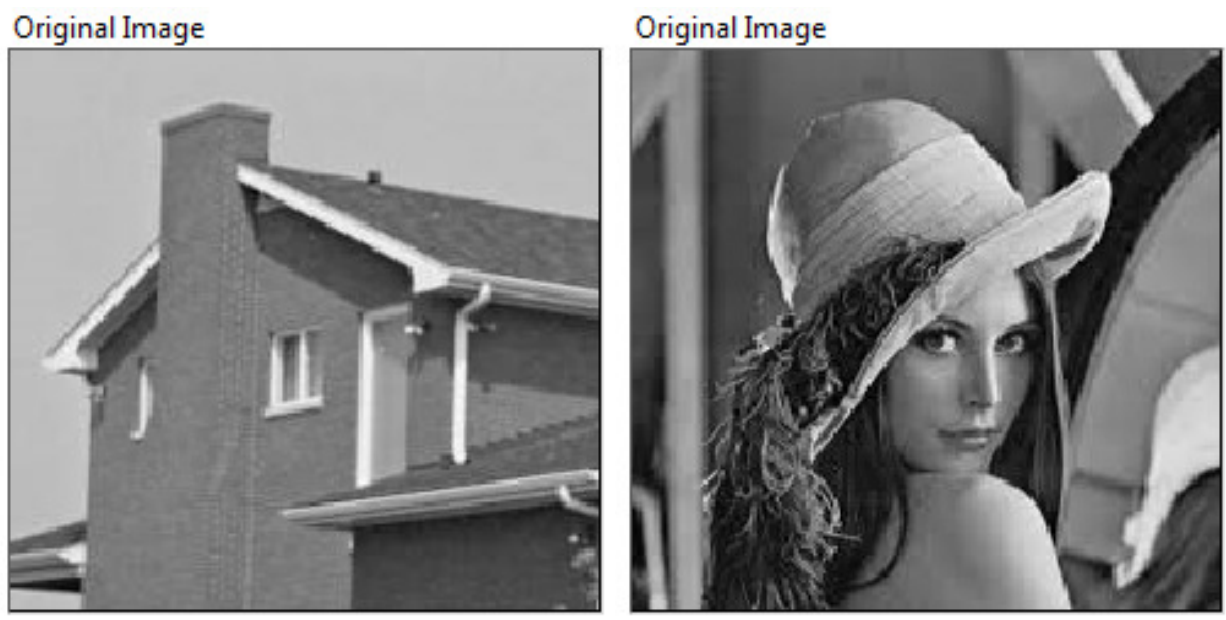
Signal \& Image Processing : An International Journal (SIPIJ) Vol.3, No.6, December 2012
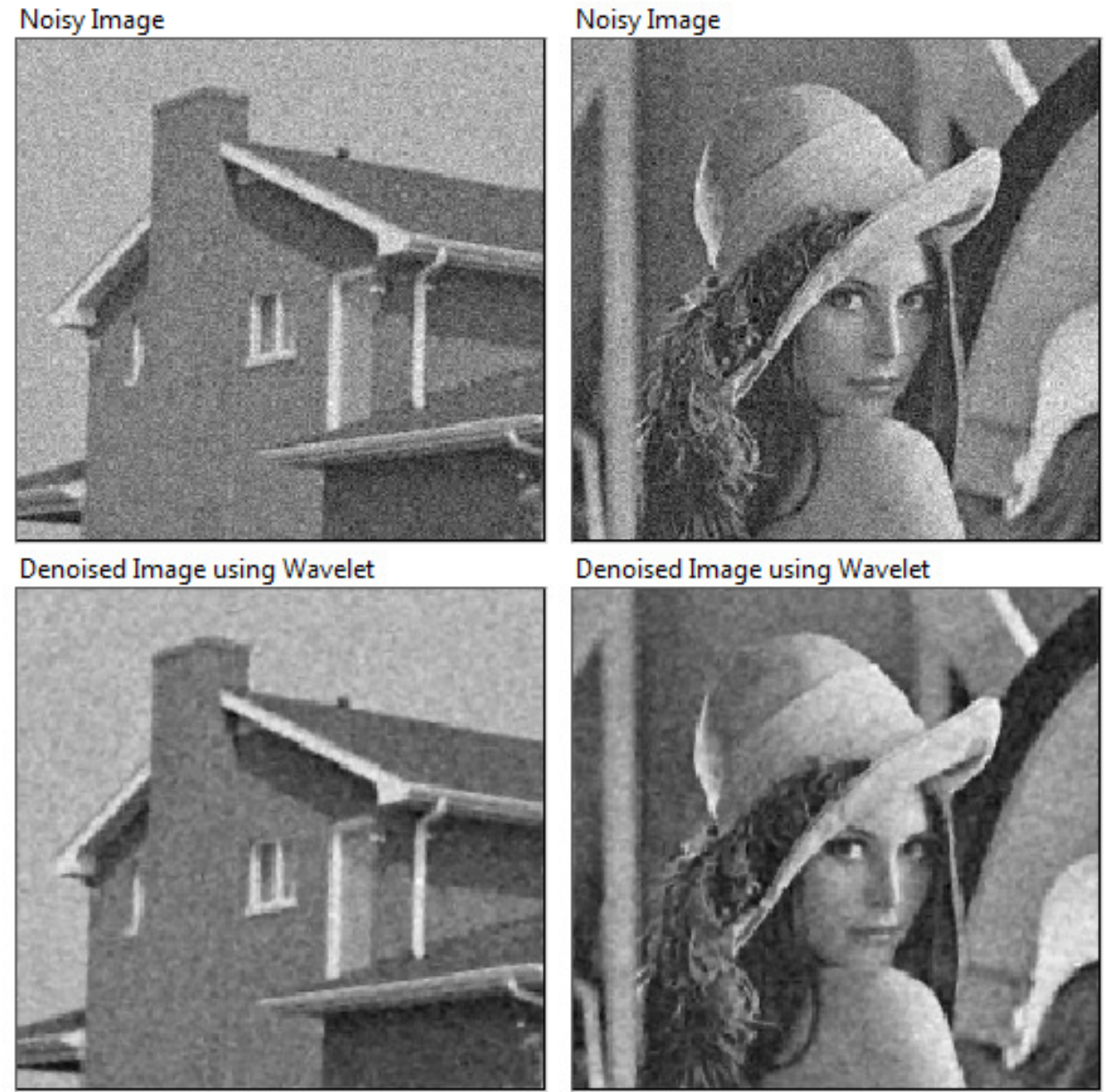

Denoised Image using Directionlet
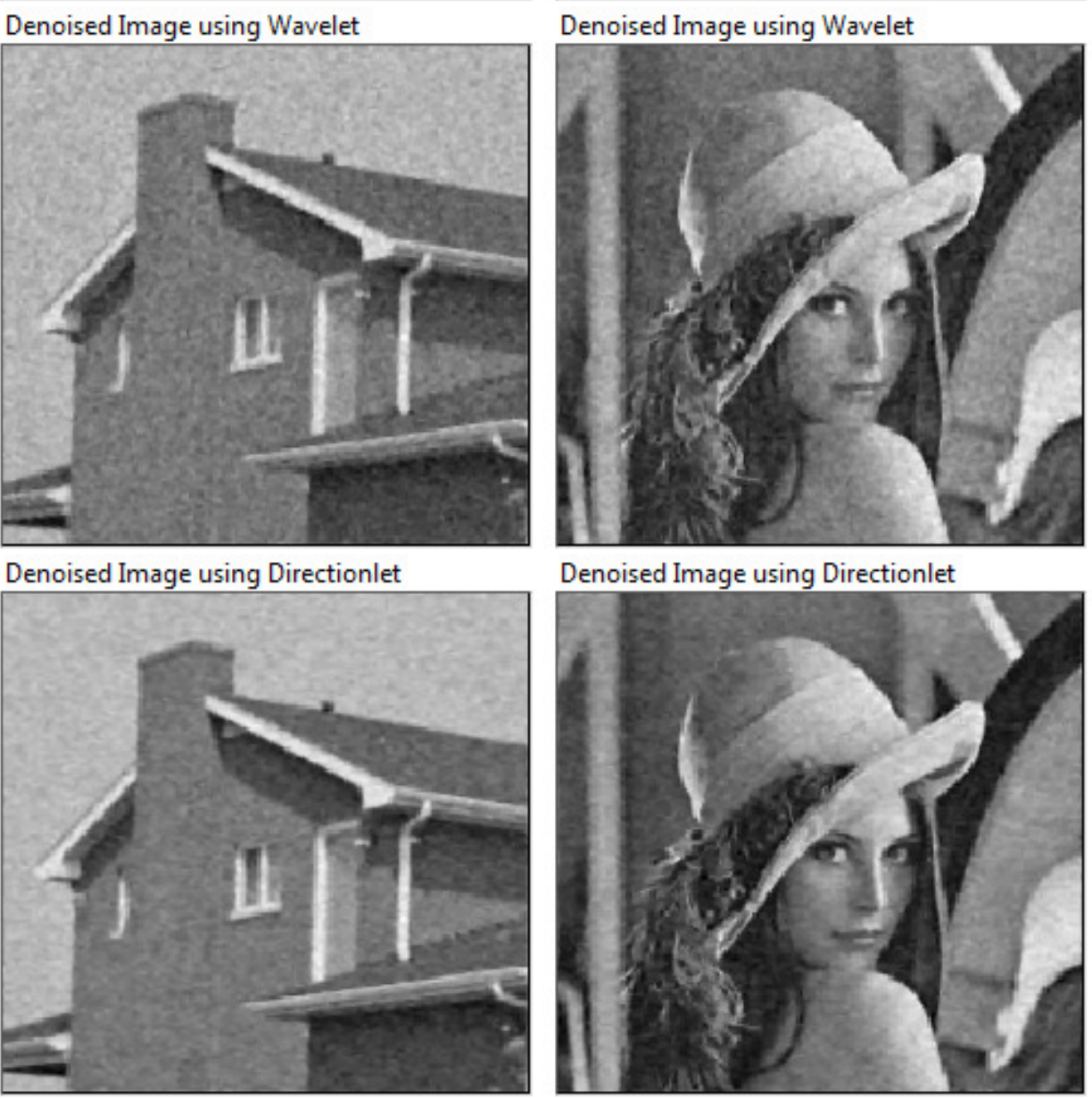

Denoised Image using Directionlet

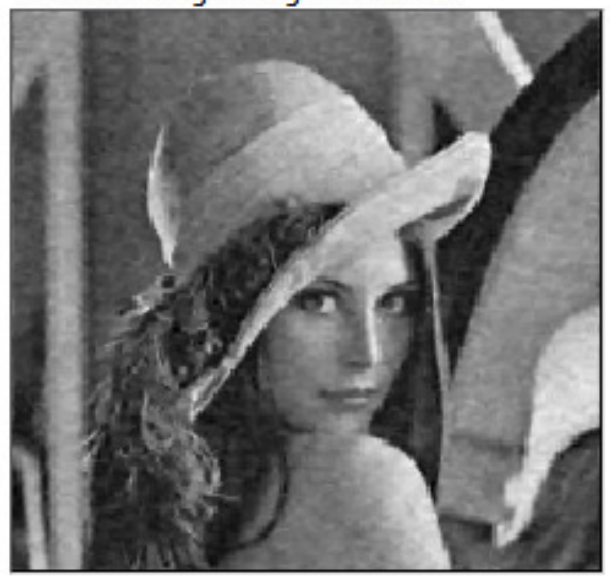

Figure.5. Image denoising using the proposed method. These images are presented in the order from top to bottom: Noise free image, noisy image with $\sigma=25$, denoised image using wavelet (soft thresholding), denoised image using directionlet.

\section{Conclusions}

In this paper a novel de-noising scheme based on multi directional and anisotropic wavelet transform called directionlet is presented. The SURE based thresholding scheme defined in 
Signal \& Image Processing : An International Journal (SIPIJ) Vol.3, No.6, December 2012

wavelet domain has been extended to the directionlet domain. Since the wavelet transform is isotropic in every scale and have limited directional capabilities, the denoising performance obtained by minimizing SURE in wavelet domain is poor around the discontinuities in the images. To overcome these limitations, we extend SURE based thresholding scheme to the directionlet domain. Since the directionlets possess spatial anisotropy and better directional capabilities, directionlet-based SURE results in visually appealing denoised results, with improved PSNR values. Since the directionlets have vanishing moments along only two directions, the dominant local directions in the image must be identified first. This was carried out by computing the directional map of the segmented image. The proposed method suitably adjusts the transform directions based on dominant directions of each segment of image and computes the threshold using SURE risk. We show by simulations that minimizing SURE in the directionlet domain results in better denoising performance when compared to minimizing it in the wavelet domain. Future work is to find out a faster method to identify the dominant local directions in an image and applying more effective threshold schemes in directionlet domain.

\section{REFERENCES}

[1] Rafeal. C . Gonzalez and Richard E. Woods, "Digital image processing", Addison Wesley Longman, 1999.

[2] I. Daubechies "The Wavelet transform, time frequency localization and signal analysis, IEEE Trans. Inform. Theory Vo1.36, pp.961-100S, Sep. 1990.

[3] M.Vetterii and C.Harley "Wavelets and filter banks: theory and design", IEEE Trans. Signal Processing Vo1.40, No.9, pp.2207-2232, Dec.1993.

[4] D. L. Donoho and I. M. Johnstone, Ideal spatial adaptation via wavelet shrinkage, Biometrika, vol. 81,pp. 425-455, 1994.

[5] D. L. Donoho and I. M. Johnstone, "Adapting to unknown smoothness via wavelet shrinkage," Journal of the American Statistical Assoc., vol. 90, no. 432, pp. 1200-1224, December 1995.

[6] E. L. Pennec and S. Mallat, "Sparse geometrical image approximation with bandelets," (2005). IEEE International Conference on Image Processing (ICIP2005)

[7] D. L. Donoho, "Wedgelets: Nearly-minimax estimation of edges," Ann. Statist., vol. 27, pp. 859-897, 1999.

[8] E. J. Cand 'es and D. L. Donoho, "Curvelets - a surprisingly effective non adaptive representation for objects with edges," in Curve and Surface Fitting, A. Cohen, C. Rabut, and L. L. Schumaker, Eds. Saint-Malo: Vanderbilt University Press, 1999.

[9] M.N.Do and Vetterli, "The contourlet transform: an efficient directional multiresolution image representation", IEEE Trans. Image Processing Vo1.12, pp. 2091-2106, Dec. 2004.

[10] Duncan.D.Y.Po and Min.N.Do, "Dierctional multiscale modeling of images using the contourlet transform", IEEE Trans. lmage Processing VoI.1S, pp.161O-1620, June 2006.

[11] Ramin Eslami and Hyder Radha, "Translation Invariant Contourlet transform and its application to Image Denoising", IEEE Trans. Image Processing VoI.1S, No.1l, pp. 3362-3374, Nov 2006.

[12] G. Liu X. Zeng Y. Liu, "Image denoising by random walk with restart Kernel and non-sub-sampled contourlet transform”, IET Signal Process., Vol. 6, Iss. 2, pp. 148-158, April 2012

[13] R.H.Bamberger and M.J.T.Smith, "A filter bank for the directional decomposition of images: Theory and Design", IEEE Trans. Signal Processing Vo1.40, No.4, pp.882-893, April 1992.

[14] D.J.Burt and E.H.Adelson, "The Laplacian pyramid as a compact image code", IEEE Trans. Commun. Vol.31, No.4, pp.532-S40, April 1983.

[15] Vladan Velisavljevic, Baltasar Beferull-Lozano, Martin Vetterly and Pier Luigi Dragotti. "Directionlets: Anisotropic Multi directional Representation with Separable Filtering", IEEE Transactions. Image processing, Vol 15 Issue 7, pp.1916-1933, July 2006

[16] V. Vladan, B. Beferull-Lozano, and M. Vetterli, "Space-frequency quantization for image compression with directionlets," IEEE Trans.Image Processing, vol. 16, no. 7, pp. 1761-1773, July 2007.

[17] Donoho D L. De-noising by soft-thresholding. IEEE Trans Inform Theory, Vol. 1, No. 2, pp. 613-618, Dec. 1995.

[18] C. Stein, "Estimation of the mean of a multivariate normal distribution," Ann. Statist., vol.9, pp. 1135-1151, 1981. 
Signal \& Image Processing : An International Journal (SIPIJ) Vol.3, No.6, December 2012

[19] Xiao-Ping Zhang and Mita D. Desai, Adaptive denoising based on SURE risk, IEEE Signal Processing Letters 5,1998, no. 10, 265-267

[20] D. Van De Ville and M. Kocher, "Nonlocal means with dimensionality reduction and SURE-based parameter selection," IEEE Trans. Image Process., vol. 20, no. 9, pp. 2683-2690, Sep. 2011.

[21] D. L. Donoho and I. M. Johnstone, "Adapting to unknown smoothness via wavelet shrinkage," J. Amer. Statist. Assoc., vol. 90, no. 432, pp. 1200-1224, Dec. 1995.

[22] S. G. Chang, B. Yu, and M. Vetterli, "Adaptive wavelet thresholding for image denoising and compression,” IEEE Trans. Image Process., vol. 9, no. 9, pp. 1135-1151, Sep. 2000.

[23] S. G. Chang, B. Yu, and M. Vetterli, "Spatially adaptive wavelet thresholding with context modeling for image denoising," IEEE Trans. Image Process., vol. 9, no. 9, pp. 1522-1531, Sep. 2000.

[24] J. Portilla, V. Strela, M. J. Wainwright, and E. P. Simoncelli, "Image denoising using scale mixtures of gaussians in the wavelet domain," IEEE Trans. Image Process., vol. 12, no. 11, pp. 1338-1351, Nov. 2003.

[25] G. Chen W.-P. Zhu W. Xie, “Wavelet-based image denoising using three scales of dependency” IET Image Process., Vol. 6, Iss. 6, pp. 756-760, August 2012

\section{Authors}

Sethunadh $\mathbf{R}$ received his $\mathrm{B}$. Tech degree in electronics \& communication engineering from University of Kerala and M. Tech degree in digital electronics with first rank from Cochin University of Science and Technology, Cochin, India. He started his career in airworthiness certification at Indian Defence Research and Development Organisation (DRDO) in 1995. Since 1997 he has been with Vikram Sarabhai Space Centre, Indian Space Research Organisation (ISRO), engaged in launch vehicle development program. He is also pursuing his $\mathrm{Ph}$. D at Department of

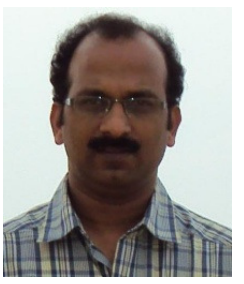
Electronics, Cochin University of Science and Technology. His research interest includes image processing, computer vision, signal processing, test automation etc.

Dr.Tessamma Thomas received her M.Tech. and Ph.D from Cochin University of Science and Technology, Cochin-22, India. At present she is working as Professor in the Department of Electronics, Cochin University of Science and Technology. She has to her credit more than 100 research papers, in various research fields, published in International and National journals and conferences. Her areas of interest include digital signal / image processing, bio medical image processing, super resolution, content based image retrieval, genomic signal processing, etc.

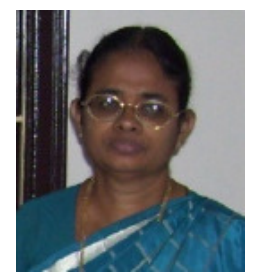

